\title{
Prognostic models for intracerebral hemorrhage: systematic review and meta- analysis
}

Tiago Gregório ${ }^{1,2^{*}}$ D, Sara Pipa ${ }^{1}$, Pedro Cavaleiro ${ }^{3}$, Gabriel Atanásio ${ }^{1}$, Inês Albuquerque ${ }^{4}$, Paulo Castro Chaves ${ }^{4,5,6}$ and Luís Azevedo ${ }^{7}$

\begin{abstract}
Background: Prognostic tools for intracerebral hemorrhage $(\mathrm{ICH})$ patients are potentially useful for ascertaining prognosis and recommended in guidelines to facilitate streamline assessment and communication between providers. In this systematic review with meta-analysis we identified and characterized all existing prognostic tools for this population, performed a methodological evaluation of the conducting and reporting of such studies and compared different methods of prognostic tool derivation in terms of discrimination for mortality and functional outcome prediction.
\end{abstract}

Methods: PubMed, ISI, Scopus and CENTRAL were searched up to 15th September 2016, with additional studies identified using reference check. Two reviewers independently extracted data regarding the population studied, process of tool derivation, included predictors and discrimination (c statistic) using a predesignated spreadsheet based in the CHARMS checklist. Disagreements were solved by consensus. C statistics were pooled using robust variance estimation and meta-regression was applied for group comparisons using random effect models.

Results: Fifty nine studies were retrieved, including 48,133 patients and reporting on the derivation of 72 prognostic tools. Data on discrimination (c statistic) was available for 53 tools, 38 focusing on mortality and 15 focusing on functional outcome. Discrimination was high for both outcomes, with a pooled c statistic of 0.88 for mortality and 0.87 for functional outcome. Forty three tools were regression based and nine tools were derived using machine learning algorithms, with no differences found between the two methods in terms of discrimination $(p=0.490)$. Several methodological issues however were identified, relating to handling of missing data, low number of events per variable, insufficient length of follow-up, absence of blinding, infrequent use of internal validation, and underreporting of important model performance measures.

Conclusions: Prognostic tools for ICH discriminated well for mortality and functional outcome in derivation studies but methodological issues require confirmation of these findings in validation studies. Logistic regression based risk scores are particularly promising given their good performance and ease of application.

Keywords: Intracerebral hemorrhage, Prognosis, Clinical prediction rules, Mortality, Morbidity

\footnotetext{
* Correspondence: tiago.gregorio@chvng.min-saude.pt

${ }^{1}$ Department of Internal Medicine, Vila Nova de Gaia Hospital Cente, Rua

Conceição Fernandes, 4434-502 Vila Nova de Gaia, Portugal

${ }^{2}$ Stroke Unit, Vila Nova de Gaia Hospital Center, Rua Conceição Fernandes,

4434-502 Vila Nova de Gaia, Portugal

Full list of author information is available at the end of the article
}

(c) The Author(s). 2018 Open Access This article is distributed under the terms of the Creative Commons Attribution 4.0 International License (http://creativecommons.org/licenses/by/4.0/), which permits unrestricted use, distribution, and reproduction in any medium, provided you give appropriate credit to the original author(s) and the source, provide a link to the Creative Commons license, and indicate if changes were made. The Creative Commons Public Domain Dedication waiver (http://creativecommons.org/publicdomain/zero/1.0/) applies to the data made available in this article, unless otherwise stated. 


\section{Background}

Intracerebral hemorrhage ( $\mathrm{ICH})$ is a major cause of death and disability, with an incidence rate of 24.6 per 100,000 person-years and a fatality rate of $40 \%$. After such event, only $12-39 \%$ of patients regain independence [1]. Contrary to ischemic stroke, medical care for $\mathrm{ICH}$ remains mostly supportive, and few interventions clearly demonstrated benefit in this population [2, 3]. Several prognostic tools have been proposed for mortality and functional outcome prediction in $\mathrm{ICH}$. These tools are potentially useful for ascertaining prognosis, facilitating communication between clinicians, characterizing and selecting patients for interventions, and for benchmarking purposes in healthcare delivery $[2,4]$.

The aim of this study was to systematically identify, assess and review the methodological conduct and reporting of studies deriving prognostic tools for the risk of death and/or functional recovery after ICH and to evaluate their overall discrimination according to the method of derivation and type of outcome.

\section{Methods}

We have designed, developed and reported our systematic review and meta-analysis in accordance with recommendations from the Cochrane Prognosis Methods Group [5] and the PRISMA [6] and MOOSE [7] guidelines. For this purpose, we searched PubMed, ISI Web of Knowledge, Scopus, and CENTRAL for all studies reporting the derivation of prognostic tools for predicting death and/or functional recovery after non-traumatic $\mathrm{ICH}$, using the broad and sensitive search query reported Additional file 1. The search included articles from database inception to 15th September 2016, with additional articles identified from reference checking. No language restrictions were applied. There is no protocol available.

\section{Study selection and inclusion criteria}

Articles were included if they met the following criteria: 1) were human studies; 2) were original articles; 3 ) were adult studies ( $\geq 18$ years); 4) did not consist of case reports/ case series; 5) enrolled non-traumatic ICH patients; 6) were prognostic studies; 7) described the application of a prognostic tool; and 8) were derivation studies. Studies involving traumatic and/or extra-axial bleedings were excluded. Study selection was performed using a two-step process. In the first step (screening), all abstracts were reviewed by two authors independently applying the inclusion criteria. This process was repeated in the second step again by two authors working independently, applying the same criteria to the full text of remaining studies. Disagreements were resolved by consensus.

\section{Quality assessment, data extraction, analysis and} reporting

To inform quality assessment and data extraction from individual studies, two reviewers independently applied a spreadsheet based in the CHARMS checklist [5] to the included studies, gathering information on the following aspects of prognostic tool derivation: 1) population, sampling and source of data; 2) outcome timing and definition; 3) number and type of predictors; 4) number of patients and events 5) handling of missing data; 6) method for tool derivation and 7) prognostic tool performance.

Prognostic tool performance was evaluated by determining its discriminatory capacity, i.e., its ability to determine which patients will suffer the outcome of interest. As a measure of this, we retrieved the c-statistic along with its $95 \%$ confidence interval (CI). For studies not reporting any of these parameters, we obtained them by recreating the receiver operating characteristic (ROC) curve from reported probability distributions; for studies reporting the c-statistic but not its confidence interval, we calculated the later using the method reported by Hanley and McNeil [8], where the number of outcomes was available. Standard errors were derived from the respective CIs.

Given the fact that some authors derived more than one tool from the same sample population, we pooled c-statistics using robust variance estimation (RVE) to account for dependent effects, according to Tanner-Smith et al. [9]. Specifically, we assumed correlated effect sizes and used a random effects model with inverse variance weights to estimate the overall mean c-statistic and mean c-statistics for mortality prediction tools, functional outcome prediction tools, logistic regression based tools, and machine learning algorithms. Univariate meta-regression was used to compare these groups and $p$ values $<0.05$ were considered significant. Due to the nature of the meta-analytical technique used, heterogeneity statistics such as Q-statistic and I-square are not recommended, according to Tanner-Smith et al. [9]. However, the I2 statistic is reported for illustrative purposes. Statistical analysis was performed using specific macros [9] designed for $\mathrm{R}$ and $\mathrm{SPSS}^{\bullet}$ statistics v 24.0.

\section{Results}

Figure 1 depicts the study selection procedure. The search query retrieved 15,613 references: after the screening step, there were 263 references left for full text review. The second step removed an additional 207 references, leaving us with 56 studies reporting the derivation of at least one prognostic tool. Three additional studies were identified through reference check, which led to the final number of 59 studies involving 48,133 patients. Nine studies reported the derivation of more 


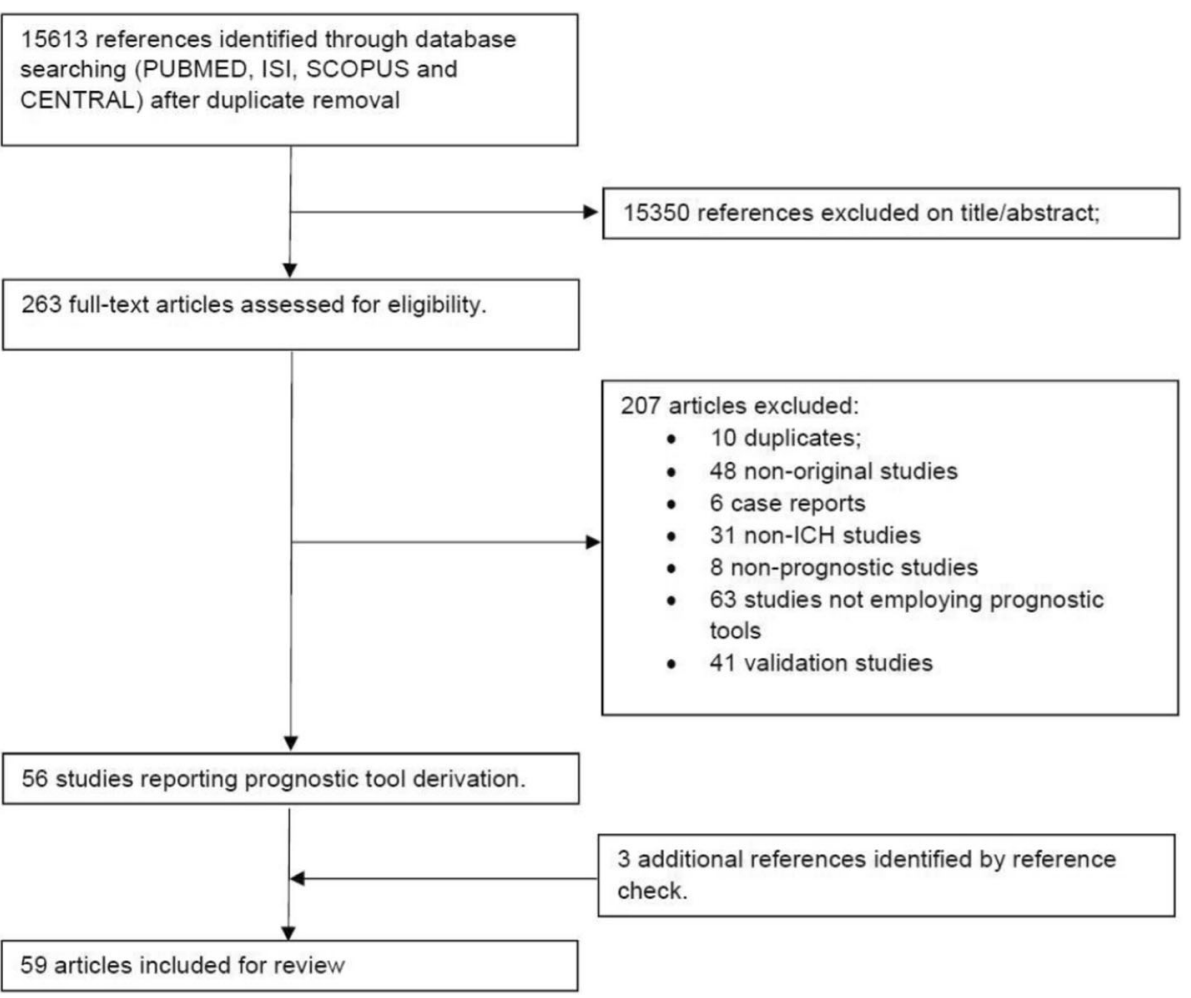

Fig. 1 Study selection flow chart

than one prognostic tool, so the total number of prognostic tools analyzed was 72 . The summary description of these tools is depicted in Table 1.

\section{Population, sampling and source of data}

The source population from which the patients were recruited for prognostic tool derivation consisted on primary/spontaneous ICH patients for all tools except two $[10,11]$, which focused on arteriovenous malformation related hemorrhages (Table 1). However, several studies included further specifications for patient inclusion namely age [12], cerebral amyloid related angiopathy [13], deep location [14-17], lobar location [18], supratentorial bleeds [16, 19-33], presence of intraventricular hemorrhage [16, 34, 35], African ethnicity [36], non-comatose patients [22, 37], comatose patients [38], medically treated patients $[22-25,27,33,38-40]$, surgically treated patients [21], oral anticoagulant related bleeds [41], hypertensive patients [19, 36], and dialysis patients [40]. The majority of studies $(n=40)$ recruited patients from hospitals or emergency rooms [10-14, 16$22,26,29-31,36,40-61]$ but nine studies recruited patients from intensive care units $[24,28,32,33,35,38$, 62-64], three studies recruited patients from stroke units [34, 37, 65], six studies recruited patients from neurology/neurosurgery departments $[15,25,27,39,66$, 67], and one study recruited patients from both an intensive care unit and a stroke unit [23]. Most prognostic tools were derived from cohort studies, with the exceptions being registries $[29,30,43,51,64]$, randomized clinical trial data [14, 42], case-control studies [26], and administrative databases [59]. Thirteen studies were multicentric $[13,24,29,30,35-37,42,43,51,55,59$, 62], with two studies involving more than two countries $[42,55]$. The sampling method was not reported or unclear for 18 studies [12, 15, 17, 21, 23, 24, 26, 29, 30, 32, $36,42,54,61,63,66-68]$, being consecutive for all others.

\section{Outcome timing, definition and assessment}

Of the 72 prognostic tools included in this review 46 focused on mortality [12, 21, 23, 24, 26-36, 38-41, 44, 45, $48-50,53,54,56,58-61,63-68], 19$ focused on morbidity $[10,11,15,17-20,22,25,37,42,43,47,51,52,55$, 57], and seven were derived for a combined outcome (mortality plus morbidity) [13, 14, 16, 46, 62].

Mortality prediction was mostly attempted at hospital discharge or 1 month (Table 1); exceptions to this rule were the studies by Grellier [66], Celik [68], Lei [13], Cho [14], and Galbois [38], which analyzed death at 2 days, 10 days, 3 months, 6 months, and ICU discharge respectively. Interestingly, Galbois focused on brain death rather than the general concept of mortality used in other studies. Functional status prediction was more 
Table 1 Summary description of prognostic tools

\begin{tabular}{|c|c|c|c|c|c|c|}
\hline Author & Year & Population & Tool & Timing & Variables & AUC (SE) \\
\hline \multicolumn{7}{|c|}{ Mortality prediction tools } \\
\hline Alsina [24] & 2014 & $\begin{array}{l}\text { Supratentorial ICH } \\
\text { not submitted } \\
\text { to surgery }\end{array}$ & Equation & 30 days & $\begin{array}{l}\text { IVH, hematoma size, } \\
\text { and midline shift. }\end{array}$ & $0.933(0.029)$ \\
\hline Berwaerts [41] & 2000 & $\begin{array}{l}\text { Oral anticoagulant } \\
\text { related ICH }\end{array}$ & Equation & Discharge & $\begin{array}{l}\text { Hematoma diameter } \\
\text { and CT signs of ischemia. }\end{array}$ & - \\
\hline Bhatia [65] & 2013 & Primary $\mathrm{ICH}$ & Equation & Discharge & $\begin{array}{l}\text { GCS, hematoma size, IVH,and } \\
\text { ventilatory requirement. }\end{array}$ & $0.822(0.033)$ \\
\hline Broderick [44] & 1993 & Spontaneous ICH & Equation & 30 days & GCS, hematoma size. & $0.805(0.036)$ \\
\hline Broderick' [44] & 1993 & Spontaneous ICH & Equation & 30 days & $\begin{array}{l}\text { Hematoma size, IVH volume, } \\
\text { GCS, and surgery. }\end{array}$ & - \\
\hline Celik [68] & 2014 & Spontaneous ICH & ANN & 10 days & $\begin{array}{l}\text { Age, gender, hypertension, } \\
\text { diabetes, smoking, mean blood } \\
\text { pressure, Scandinavian Stroke } \\
\text { Scale score, pulse pressure, } \\
\text { localization of hemorrhage } \\
\text { (including infratentorial), volume } \\
\text { of hemorrhage, ventricular } \\
\text { drainage, and midline shift. }\end{array}$ & - \\
\hline Cerillo [21] & 1981 & $\begin{array}{l}\text { Operated } \\
\text { supratentorial ICH }\end{array}$ & Equation & Discharge & $\begin{array}{l}\text { Age, mode of onset, site of } \\
\text { hemorrhage, level of } \\
\text { consciousness, time from } \\
\text { onset to surgery, congestive } \\
\text { heart failure/coronary artery } \\
\text { disease, and diabetes/uremia. }\end{array}$ & $0.893(0.033)$ \\
\hline Chen [45] & 2011 & Nontraumatic ICH & Score & Discharge & $\begin{array}{l}\text { GCS, hematoma volume, } \\
\mathrm{IVH} \text {, and diabetes. }\end{array}$ & $0.867(0.027)$ \\
\hline Chiu [61] & 2016 & Spontaneous ICH & CART+SVM & 30 days & GCS, hematoma size. & - \\
\hline Chuang [63] & 2009 & Spontaneous ICH & Score & 30 days & $\begin{array}{l}\text { Age, GCS, hypertension, } \\
\text { glucose and dialysis } \\
\text { dependency. }\end{array}$ & $0.890(0.026)$ \\
\hline Edwards [28] & 1999 & Supratentorial ICH & ANN & Discharge & $\begin{array}{l}\text { Gender, race, hydrocephalus, } \\
\text { mean arterial pressure, pulse } \\
\text { pressure, GCS, IVH, hematoma } \\
\text { size, location (thalamic, } \\
\text { basal, lobal), cisternal } \\
\text { effacement, pineal shift, } \\
\text { hypertension, } \\
\text { diabetes, and age. }\end{array}$ & $0.984(0.020)$ \\
\hline Edwards' [28] & 1999 & Supratentorial ICH & Equation & Discharge & $\begin{array}{l}\text { Hydrocephalus, GCS, } \\
\text { gender, pineal shift }\end{array}$ & $0.919(0.043)$ \\
\hline Fogelholm [31] & 1997 & Supratentorial ICH & Equation & 28 days & $\begin{array}{l}\text { Consciousness, mean } \\
\text { arterial pressure, subarachnoid } \\
\text { spread, midline shift, } \\
\text { glucose, and vomiting. }\end{array}$ & - \\
\hline Frithz [12] & 1976 & ICH patients $<70$ years & Decision tree & Discharge & Consciousness, diastolic blood pressure. & $0.943(0.024)$ \\
\hline Galbois [38] & 2013 & $\begin{array}{l}\text { Spontaneous comatose } \\
\text { ICH not submitted } \\
\text { to surgery }\end{array}$ & Score & ICU stay & Brainstem reflexes, swirl sign. & $0.850(0.050)$ \\
\hline Galbois' [38] & 2013 & $\begin{array}{l}\text { Spontaneous comatose } \\
\text { ICH not submitted } \\
\text { to surgery }\end{array}$ & Score & ICU stay & Corneal reflexes, swirl sign. & $0.840(0.051)$ \\
\hline Grellier [66] & 1983 & Spontaneous ICH & Score & 2 days & $\begin{array}{l}\text { Age, gender, consciousness } \\
\text { (normal, changed, coma), } \\
\text { CV risk factors (alcohol, tobacco, } \\
\text { hypertension, dyslipidemia, } \\
\text { CV disease), and ICH location } \\
\text { (infratentorial, thalamic, } \\
\text { internal capsule, oval center, lobar). }\end{array}$ & - \\
\hline Hallevi [34] & 2009 & Primary ICH with IVH & Score & Discharge & $\mathrm{GCS}$, total volume $(\mathrm{ICH}+\mathrm{IVH})$. & $0.840^{\mathrm{a}}$ \\
\hline Hemphill [48] & 2001 & Nontraumatic $\mathrm{ICH}$ & Score & 30 days & $\begin{array}{l}\text { Age, ICH volume, infratentorial } \\
\mathrm{ICH}, \mathrm{GCS} \text {, and IVH. }\end{array}$ & $0.920(0.020)$ \\
\hline Ho [64] & 2016 & Primary ICH & Score & Discharge & $\begin{array}{l}\text { Age, creatinine, NIHSS, } \\
\text { heart disease, gender, }\end{array}$ & $0.870(0.018)$ \\
\hline
\end{tabular}


Table 1 Summary description of prognostic tools (Continued)

\begin{tabular}{|c|c|c|c|c|c|c|}
\hline Author & Year & Population & Tool & Timing & Variables & AUC (SE) \\
\hline & & & & & and systolic blood pressure. & \\
\hline Huang [40] & 2008 & $\begin{array}{l}\text { Spontaneous } \\
\text { medically treated } \\
\text { ICH in hemodialysis } \\
\text { patients }\end{array}$ & Score & 30 days & $\begin{array}{l}\text { GCS, age, and systolic } \\
\text { blood pressure. }\end{array}$ & $0.745(0.048)$ \\
\hline Li [50] & 2012 & Spontaneous ICH & Equation & Discharge & $\begin{array}{l}\text { Age, GCS, glucose, and } \\
\text { white blood cell count. }\end{array}$ & $0.923(0.020)$ \\
\hline$L^{\prime} i^{\prime}[49]$ & 2011 & Primary ICH & Score & Discharge & $\begin{array}{l}\text { Age, Glucose, LDH, } \\
\text { and white blood cell count. }\end{array}$ & $0.745(0.025)$ \\
\hline Lukic [33] & 2012 & $\begin{array}{l}\text { Primary supratentorial } \\
\text { medically treated ICH }\end{array}$ & Equation & Discharge & $\begin{array}{l}\text { Level of consciousness, GCS } \\
\text { verbal response, age, } \\
\text { gender, and pulse pressure. }\end{array}$ & $0.856(0.018)$ \\
\hline Lukic' [26] & 2012 & $\begin{array}{l}\text { Spontaneous } \\
\text { supratentorial ICH }\end{array}$ & ANN & Discharge & $\begin{array}{l}\text { Age, gender, pulse pressure, } \\
\text { mean arterial pressure, } \\
\text { GCS (EN/M), and consciousness. }\end{array}$ & $0.883(0.048)$ \\
\hline Lukic" [26] & 2012 & $\begin{array}{l}\text { Spontaneous } \\
\text { supratentorial ICH }\end{array}$ & Equation & Discharge & GCS, level of consciousness. & $0.819(0.030)$ \\
\hline Masé [27] & 1995 & $\begin{array}{l}\text { Primary supratentorial } \\
\text { medically treated } \mathrm{ICH}\end{array}$ & Equation & 30 days & $\begin{array}{l}\text { GCS, IVH spread, } \\
\text { and hematoma size. }\end{array}$ & - \\
\hline Parry-Jones [53] & 2013 & Spontaneous ICH & Equation & 30 days & $\begin{array}{l}\text { Age, GCS, IVH extension, } \\
\text { and hematoma volume. }\end{array}$ & $0.897(0.010)$ \\
\hline Peng [54] & 2010 & Spontaneous ICH & Random Forrest & 30 days & $\begin{array}{l}\text { Age, gender, hypertension, } \\
\text { diabetes, ischemic heart } \\
\text { disease, previous stroke, } \\
\text { anemia, dialysis dependency, } \\
\text { GCS, systolic/diastolic/mean } \\
\text { blood pressure, infratentorial } \\
\text { bleed, site of ICH, ICH volume, } \\
\text { IVH, pineal shift, hydrocephalus, } \\
\text { hemoglobin, and glucose. }\end{array}$ & $0.870(0.015)$ \\
\hline Peng' [54] & 2010 & Spontaneous $\mathrm{ICH}$ & ANN & 30 days & $\begin{array}{l}\text { Age, gender, GCS, site of ICH, } \\
\text { ICH volume, IVH, hypertension, } \\
\text { diabetes, anemia, } \\
\text { and previous stroke. }\end{array}$ & $0.810(0.020)$ \\
\hline Peng" [54] & 2010 & Spontaneous ICH & SVM & 30 days & $\begin{array}{l}\text { Age, gender, GCS, site, } \\
\text { ICH volume, IVH, hypertension, } \\
\text { diabetes, anemia, } \\
\text { and previous stroke. }\end{array}$ & $0.790(0.020)$ \\
\hline Peng"' [54] & 2010 & Spontaneous ICH & Equation & 30 days & $\begin{array}{l}\text { Anemia, age, GCS, } \\
\text { hypertension, and dialysis dependency. }\end{array}$ & $0.780(0.020)$ \\
\hline Romano [56] & 2009 & Primary ICH & Score & 30 days & $\begin{array}{l}\text { GCS, hematoma volume, } \\
\text { and intraventricular spread. }\end{array}$ & $0.915(0.026)$ \\
\hline Ruiz-Sandoval [58] & 2007 & Primary ICH & Score & Discharge & $\begin{array}{l}\text { Age, infratentorial bleed, } \\
\text { ICH size, GCS, and IVH spread. }\end{array}$ & $0.880(0.017)$ \\
\hline Safatli [60] & 2016 & Primary ICH & Score & 30 days & $\begin{array}{l}\text { GCS, infratentorial bleed, } \\
\text { and hematoma volume. }\end{array}$ & - \\
\hline Szepesi [32] & 2015 & Supratentorial ICH & Equation & 30 days & $\begin{array}{l}\text { Age, hematoma volume, } \\
\text { IVH, systolic blood pressure, } \\
\text { glucose, and potassium. }\end{array}$ & - \\
\hline Tabak [59] & 2007 & Spontaneous ICH & Equation & Discharge & $\begin{array}{l}\text { Age, creatinine, glucose, pH, } \\
\mathrm{CO2}, \mathrm{O} 2 \text {, partial thromboplastin } \\
\text { time, prothrombin time, } \\
\text { platelets, white blood cells, } \\
\text { cancer, temperature, pulse, } \\
\text { systolic blood pressure, } \\
\text { respiratory rate, } \\
\text { and altered mental status. }\end{array}$ & $0.890(0.003)$ \\
\hline Takahashi [67] & 2006 & Spontaneous ICH & CART & Discharge & $\begin{array}{l}\text { Japan Coma Scale, } \\
\text { ICH volume, and age. }\end{array}$ & $0.853(0.024)$ \\
\hline Takahashi' [67] & 2006 & Spontaneous ICH & Equation & Discharge & $\begin{array}{l}\text { Japan Coma Scale, } \\
\text { temperature, infratentorial } \\
\text { bleed, and ICH volume. }\end{array}$ & $0.810(0.033)$ \\
\hline Tshikwela [36] & 2012 & $\begin{array}{l}\text { Black hypertensive } \\
\text { primary ICH }\end{array}$ & Score & Discharge & $\begin{array}{l}\text { GCS, ICH volume, } \\
\text { left hemisphere involved. }\end{array}$ & - \\
\hline
\end{tabular}


Table 1 Summary description of prognostic tools (Continued)

\begin{tabular}{|c|c|c|c|c|c|c|}
\hline Author & Year & Population & Tool & Timing & Variables & AUC (SE) \\
\hline Tshikwela' [36] & 2012 & $\begin{array}{l}\text { Black hypertensive } \\
\text { primary ICH }\end{array}$ & Score & Discharge & Gender, GCS, midline shift. & - \\
\hline Tuhrim [23] & 1999 & $\begin{array}{l}\text { Primary supratentorial } \\
\text { ICH managed medically }\end{array}$ & Equation & 30 days & $\begin{array}{l}\mathrm{GCS}, \mathrm{ICH} \text { volume, pulse } \\
\text { pressure, hydrocephalus, } \\
\text { and IVH volume. }\end{array}$ & - \\
\hline Tuhrim' [30] & 1991 & Supratentorial ICH & Equation & 30 days & $\begin{array}{l}\text { Hematoma size, IVH, } \\
\text { GCS, pulse pressure, } \\
\text { and IVH }{ }^{\mathrm{a} G C S} \text { interaction. }\end{array}$ & $0.900(0.027)$ \\
\hline Tuhrim" [29] & 1988 & Supratentorial hemorrhage & Equation & 30 days & $\begin{array}{l}\text { GCS score, hematoma } \\
\text { size, and pulse pressure. }\end{array}$ & $0.892(0.042)$ \\
\hline Ziai [35] & 2015 & Primary ICH with IVH & Score & Discharge & $\begin{array}{l}\text { Temperature, glucose, } \\
\text { intracranial pressure, } \\
\text { and Do-Not-Resuscitate orders }\end{array}$ & $0.850(0.030)$ \\
\hline Zis [39] & 2014 & Non-operated primary ICH & Score & 30 days & $\begin{array}{l}\text { GCS, ICH size, INR, IVH } \\
\text { spread, and } \\
\text { infratentorial location. }\end{array}$ & $0.920(0.023)$ \\
\hline \multicolumn{7}{|c|}{ Functional outcome prediction tools } \\
\hline Appelboom [10] & 2012 & AVM related $\mathrm{ICH}$ & Score & 3 months & $\begin{array}{l}\text { Age, IVH, infratentorial } \\
\text { bleed, GCS, and hematoma size. }\end{array}$ & $0.914(0.039)$ \\
\hline Creutzfeld [47] & 2011 & Primary ICH & Equation & Discharge & $\begin{array}{l}\text { Age, GCS, heart rate, } \\
\text { mass effect, IVH, premorbid } \\
\text { level of function, and } \\
\text { systolic blood pressure. }\end{array}$ & $0.930(0.014)$ \\
\hline Flemming [18] & 2001 & $\begin{array}{l}\text { Lobar primary } \\
\text { supratentorial ICH }\end{array}$ & Tree based model & Discharge & GCS, septum pellucidum shift. & $0.890(0.045)$ \\
\hline Flemming' [18] & 2001 & $\begin{array}{l}\text { Lobar primary } \\
\text { supratentorial ICH }\end{array}$ & Tree based model & Discharge & ICH size, GCS, and time to presentation. & $0.921(0.032)$ \\
\hline Hallevy [25] & 2002 & $\begin{array}{l}\text { Primary supratentorial } \\
\text { medically treated ICH }\end{array}$ & Score & Discharge & $\begin{array}{l}\text { Age, limb paresis, level of } \\
\text { consciousness, mass effect, } \\
\text { hematoma size, and } \\
\text { intraventricular extension. }\end{array}$ & $0.897(0.023)$ \\
\hline Ji [51] & 2013 & Spontaneous $\mathrm{ICH}$ & Score & 1 year & $\begin{array}{l}\text { Age, NIHSS, GCS, glucose, } \\
\text { infratentorial bleed, } \\
\text { ICH volume, and IVH. }\end{array}$ & $0.836(0.009)$ \\
\hline Lisk [22] & 1994 & Primary supratentorial $<24 \mathrm{~h}$ & Equation & Discharge or 30 days & $\begin{array}{l}\text { Age, GCS, hemorrhage } \\
\text { volume, and gender. }\end{array}$ & - \\
\hline Lisk' [22] & 1994 & $\begin{array}{l}\text { Primary supratentorial } \\
<24 h, G C S>9 \text {, no surgery }\end{array}$ & Equation & Discharge or 30 days & $\begin{array}{l}\text { Age, hemorrhage diameter, } \\
\text { and ventricular extension. }\end{array}$ & - \\
\hline Neidert [11] & 2016 & AVM related $\mathrm{ICH}$ & Score & Unclear & $\begin{array}{l}\text { Age, GCS, hematoma size, } \\
\text { IVH, AVM size, diffuse nidus, } \\
\text { eloquence, and } \\
\text { deep venous drainage. }\end{array}$ & $0.842(0.046)$ \\
\hline Misra [15] & 1999 & Primary putaminal ICH & Equation & 3 months & $\begin{array}{l}\text { GCS, pupillary change, } \\
\text { incontinence, and location } \\
\text { of hematoma } \\
\text { (cortical, subcortical, } \\
\text { medial or lateral). }\end{array}$ & - \\
\hline Mittal [52] & 2011 & Primary $\mathrm{ICH}$ & Score & Discharge & $\begin{array}{l}\text { Age, infratentorial, ICH } \\
\text { size, GCS, cognitive } \\
\text { impairment, and FOUR score. }\end{array}$ & - \\
\hline Portenoy [20] & 1987 & $\begin{array}{l}\text { Nontraumatic } \\
\text { supratentorial } \\
\text { spontaneous ICH }\end{array}$ & Equation & Unclear & $\begin{array}{l}\text { GCS, ICH size } \\
\text { (index), and IVH spread. }\end{array}$ & - \\
\hline Poungvarin [55] & 2006 & Primary ICH & Equation & Discharge & $\begin{array}{l}\text { Fever, ICH size }>30 \\
\text { GCS, and IVH spread. }\end{array}$ & - \\
\hline Rost [57] & 2008 & Primary $\mathrm{ICH}$ & Score & 3 months & $\begin{array}{l}\text { Age, GCS, hematoma size, } \\
\text { location (infratentorial/deep/lobar), } \\
\text { and cognitive impairment. }\end{array}$ & $0.879(0.017)$ \\
\hline Shah [17] & 2005 & Thalamic hemorrhage & Equation & 3 months & $\begin{array}{l}\text { Posterolateral ICH extension, } \\
\text { Canadian Neurological Scale. }\end{array}$ & - \\
\hline Shaya [19] & 2005 & $\begin{array}{l}\text { Hypertensive } \\
\text { supratentorial ICH }\end{array}$ & Score & 6 months & $\begin{array}{l}\text { Focal neurological deficit, } \\
\text { hydrocephalus, ICH volume }\end{array}$ & - \\
\hline
\end{tabular}


Table 1 Summary description of prognostic tools (Continued)

\begin{tabular}{|c|c|c|c|c|c|c|}
\hline Author & Year & Population & Tool & Timing & Variables & AUC (SE) \\
\hline Weimar [42] & 2009 & $\begin{array}{l}\text { Patients included } \\
\text { in ICH trials }\end{array}$ & Equation & 3 months & Age, NIHSS, and level of consciousness. & $0.805(0.020)$ \\
\hline Weimar' [37] & 2006 & $\begin{array}{l}\text { Non-comatose } \\
\mathrm{ICH} \text { patients }\end{array}$ & Equation & 100 days & Age, NIHSS. & $0.861(0.029)$ \\
\hline Weimar" [43] & 2006 & Spontaneous ICH & Score & 100 days & $\begin{array}{l}\text { Age, NIHSS, and level } \\
\text { of consciousness. }\end{array}$ & $0.913(0.018)$ \\
\hline \multicolumn{7}{|c|}{ Combined outcome prediction tools } \\
\hline Cheung [46] & 2003 & Nontraumatic ICH & Score & 30 days & $\begin{array}{l}\text { IVH, subarachnoid extension, } \\
\text { pulse pressure, NIHSS, } \\
\text { and temperature. }\end{array}$ & $\begin{array}{l}- \\
-\end{array}$ \\
\hline Cheung' [46] & 2003 & Nontraumatic ICH & Score & 30 days & $\begin{array}{l}\text { Age, IVH, infratentorial bleed, } \\
\text { NIHSS, and hematoma size. }\end{array}$ & $\begin{array}{l}- \\
-\end{array}$ \\
\hline \multirow[t]{2}{*}{ Cho [14] } & \multirow[t]{2}{*}{2008} & \multirow{2}{*}{$\begin{array}{l}\text { Basal ganglia } \\
\text { hemorrhage }\end{array}$} & \multirow[t]{2}{*}{ Score } & \multirow[t]{2}{*}{6 months } & \multirow[t]{2}{*}{ GCS, ICH volume, and IVH. } & $0.897(0.033)^{b}$ \\
\hline & & & & & & $\begin{array}{l}\text { Barthel } 0.884^{\mathrm{a}} \\
\text { GOS } 0.935^{\mathrm{ac}}\end{array}$ \\
\hline \multirow[t]{2}{*}{ Godoy [62] } & \multirow[t]{2}{*}{2006} & \multirow[t]{2}{*}{ Primary $\mathrm{ICH}$} & \multirow[t]{2}{*}{ Score } & & \multirow{2}{*}{$\begin{array}{l}\text { Age, GCS, Graeb score, } \\
\text { ICH volume, and } \\
\text { APACHE2 score } \\
\text { comorbidities. }\end{array}$} & $\begin{array}{l}0.878 \\
(0.028)^{\mathrm{b}}\end{array}$ \\
\hline & & & & 6 months $^{c}$ & & $0.893(0.025)^{c}$ \\
\hline \multirow[t]{2}{*}{ Godoy' [62] } & \multirow[t]{2}{*}{2006} & \multirow[t]{2}{*}{ Primary ICH } & \multirow[t]{2}{*}{ Score } & 30 days $^{b}$ & \multirow{2}{*}{$\begin{array}{l}\text { Age, GCS, Graeb score, } \\
\text { ICH volume, and } \\
\text { APACHE2 score } \\
\text { comorbidities. }\end{array}$} & $0.869(0.029)^{\mathrm{b}}$ \\
\hline & & & & 6 months $^{c}$ & & $0.895(0.024)^{c}$ \\
\hline \multirow[t]{2}{*}{ Lei [13] } & \multirow[t]{2}{*}{2016} & \multirow[t]{2}{*}{$\begin{array}{l}\text { Cerebral amyloid } \\
\text { related ICH }\end{array}$} & \multirow[t]{2}{*}{ Score } & \multirow[t]{2}{*}{3 months } & \multirow[t]{2}{*}{$\begin{array}{l}\text { Age, IVH, midline } \\
\text { shift, and GCS. }\end{array}$} & $\begin{array}{l}0.890 \\
(0.038)^{b}\end{array}$ \\
\hline & & & & & & $0.810(0.031)^{c}$ \\
\hline \multirow[t]{2}{*}{ Stein $[16]$} & \multirow[t]{2}{*}{2010} & Supratentorial deep & Score & 30 days $^{\mathrm{b}}$ & Age, GCS, hydrocephalus, & $0.890(0.036)^{b}$ \\
\hline & & & & 6 months $^{c}$ & and $\mathrm{CL}$ V vorume & $0.848(0.056)^{c}$ \\
\hline
\end{tabular}

SE standard error, ICH intracerebral hemorrhage, IVH intraventricular hemorrhage, CT computerized tomography, GCS Glasgow Coma Scale, ANN artificial neural networks, CART classification and regression tree, SVM support vector machine, ICU intensive care unit, CV cardiovascular, NIHSS National Institute of Health Stroke Scale, LDH lactate dehydrogenase, INR International normalized ratio, AVM arteriovenous malformation, GOS Glasgow Outcome Score

${ }^{a} \mathrm{C}$-statistics were reported but standard errors were not reported, nor were the number of outcomes

${ }^{\mathrm{b}}$ Mortality

${ }^{\mathrm{c} F u n c t i o n a l ~ o u t c o m e}$

heterogeneous on the timing and method of assessment: ten tools attempted to predict functional status at discharge/ 1 month [18, 22, 25, 46, 47, 52, 55], eight tools attempted to predict at 3 months [10,13, 15, 17, 37, 42, $43,57]$, five tools attempted to predict at 6 months [14, $16,19,62]$, and one tool attempted to predict at 1 year [51]. The studies by Portenoy [20] and Neidert [11] were unclear about the time of outcome assessment. The instrument for functional outcome evaluation also differed between studies: ten studies used the modified Rankin scale $[10,11,13,16,22,25,46,47,51,55]$, six studies used the Glasgow Outcome scale [14, 18, 19, 52, 57, 62], six studies used the Barthel index [14, 15, 17, 37, 42, 43], and one study used a subjective assessment [20]. Only six studies reported blinded outcome assessment [10, 13, $37,43,51,52]$. All outcomes were binary except in the study by Shaya [19], where the outcome was ordinal.

\section{Number and type of predictors}

The number of predictors for each prognostic tool ranged from two to 20, with the mode being three (Table 2). The five most frequently included predictors were consciousness
( $n=57)$, hematoma size $(n=43)$, age $(n=38)$, intraventricular blood $(n=32)$, and the presence of comorbidities $(n=$ 16). Figure 2 stratifies the ten most frequently used variables for mortality and functional outcome prediction.

\section{Number of patients and events}

The number of included patients varied between 38 [15] and 29,775 [59] and the number of outcomes ranged from 9 [22] to 6765 [59] (Table 2), with four studies not reporting this item $[14,15,34,66]$. The event per variable (EPV) rate ranged from 1.4 [28] to 398 [59], with 21 derivations showing a rate $<10[10-12,16,21-23,28$, $29,32,41,46,52,54,61,63,68]$.

\section{Handling of missing data and loss to follow-up}

Handling of missing data was not reported or unclear in 22 studies [11, 13, 17, 19-21, 23, 25, 27, 28, 36, 39, 45, 47, 52, 60, 63-68] (Table 2). Among studies reporting this item, all of them except two used a complete case analysis, with the exceptions using a missing cathegory $[37,59]$. Two studies failed to report the number of patients lost to follow-up [10, 15]: as for the others, the 


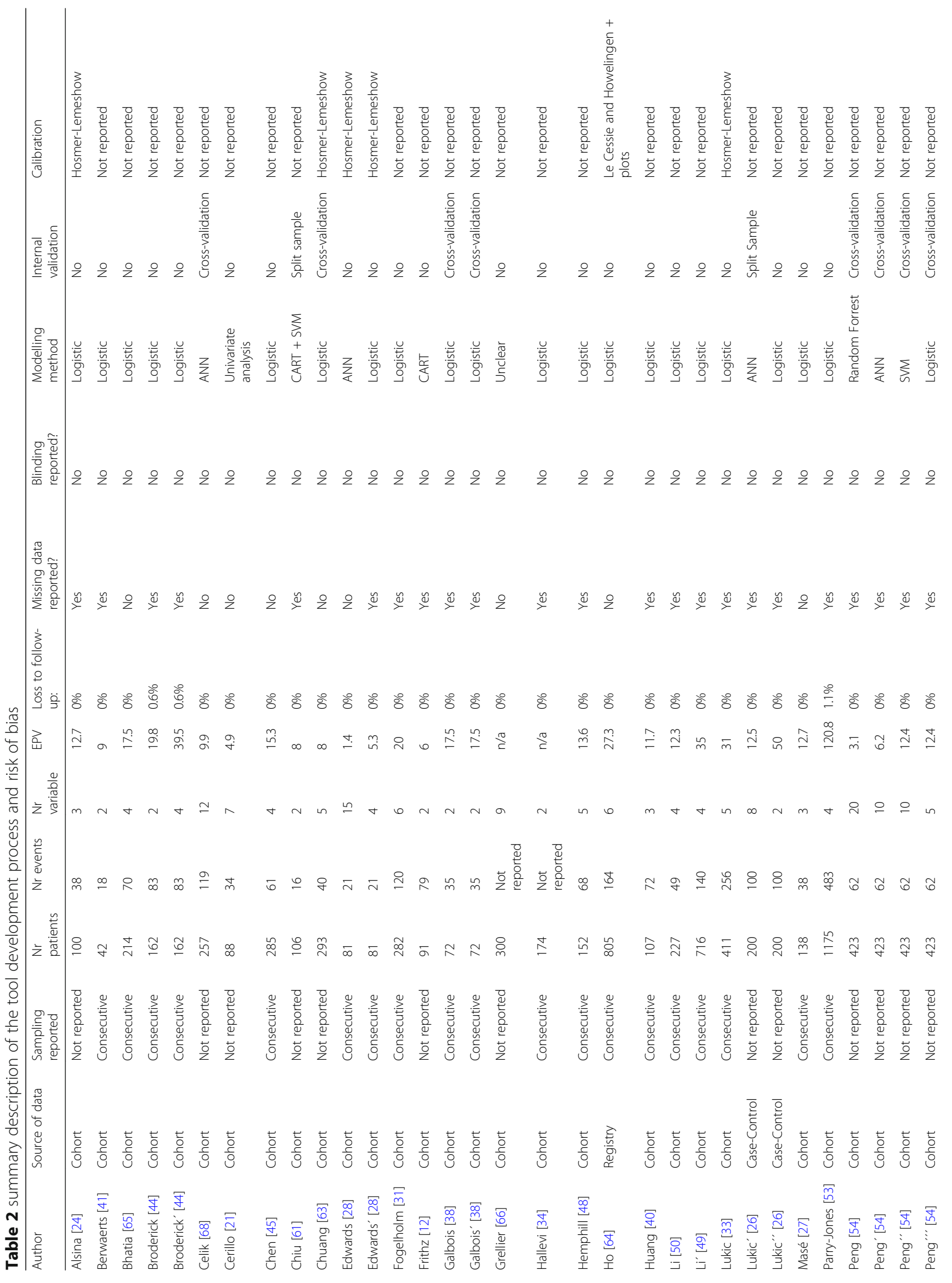




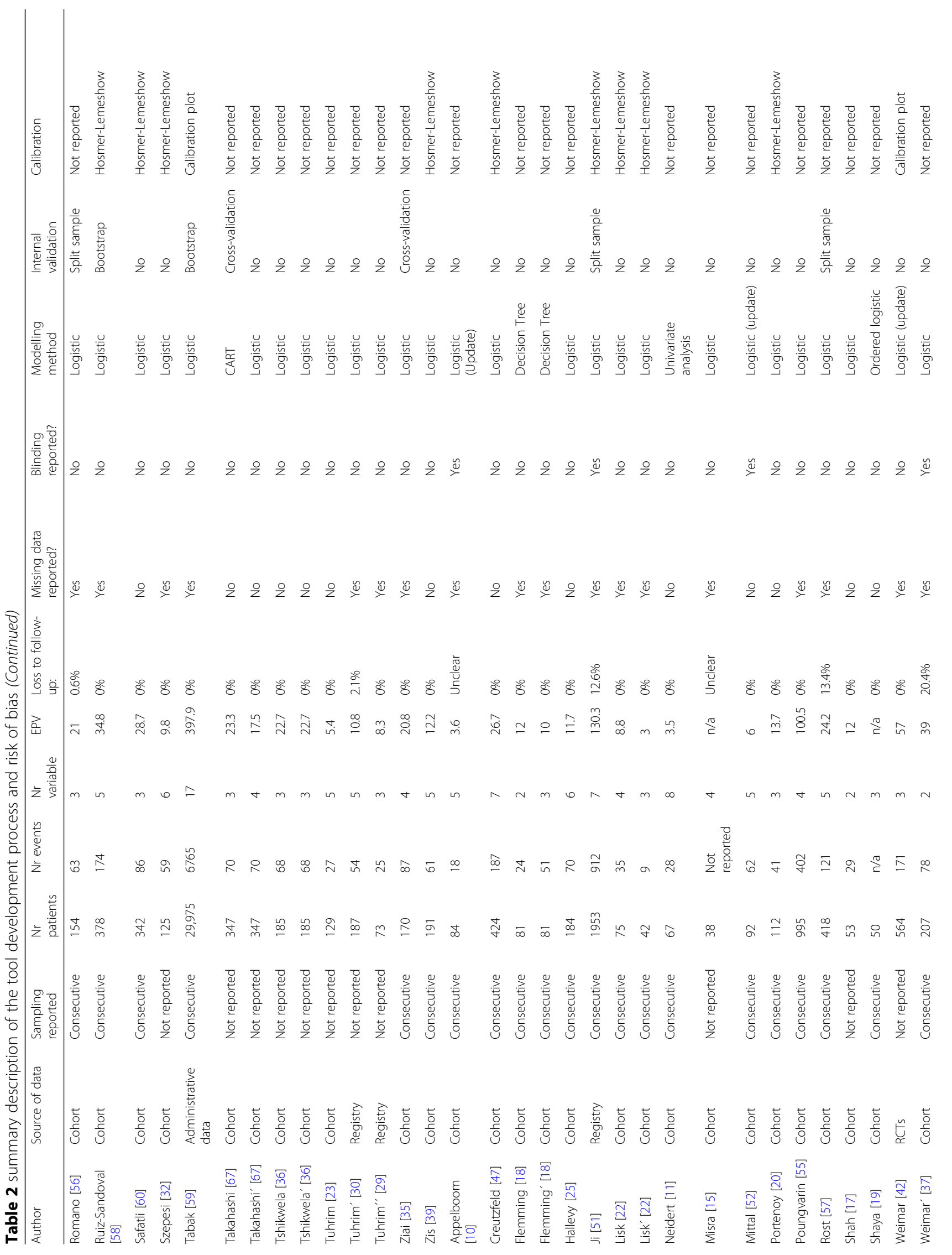




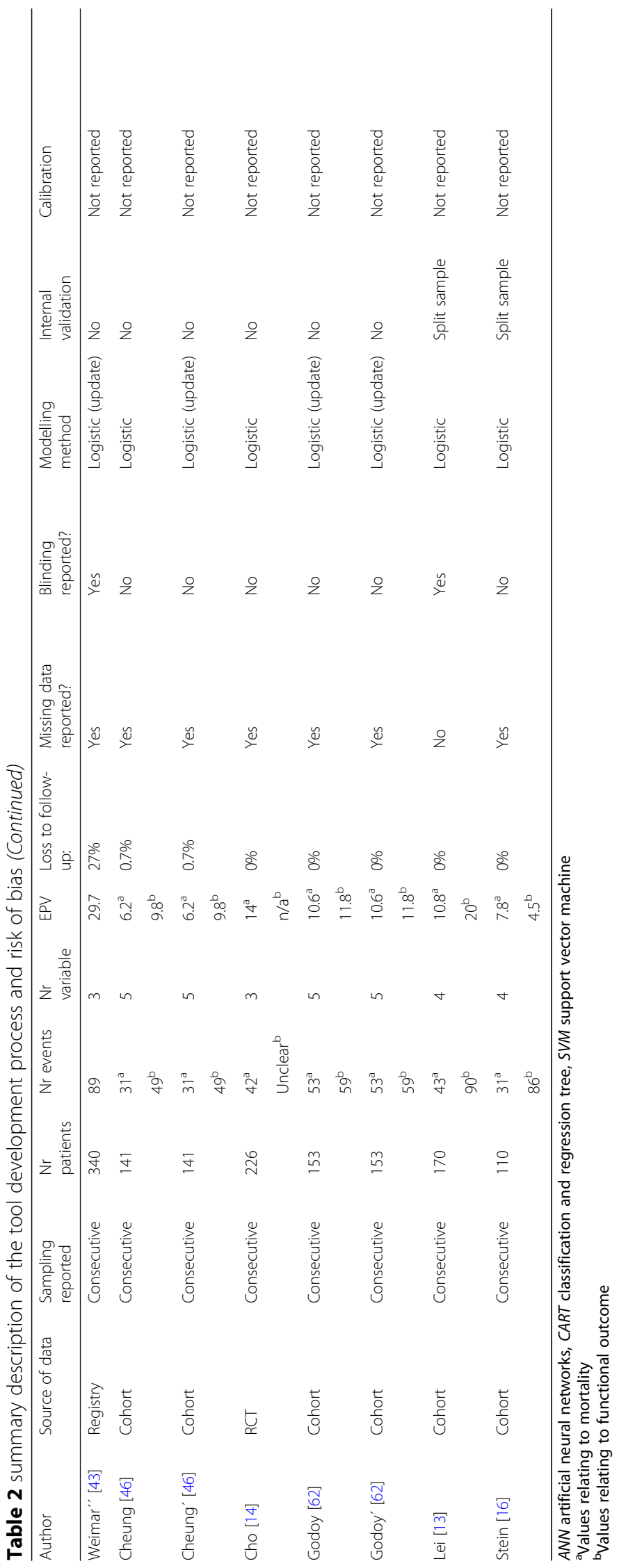


majority of them showed a $100 \%$ complete follow-up but five studies showed a loss $<5 \%$ [30, 44, 46, 53, 56], two studies showed a loss of $5-20 \%[51,57]$ and two studies showed a loss $>20 \%[37,43]$.

\section{Methods used for tool derivation}

Amongst the 72 prognostic tools encountered, 58 were regression based [10, 13-17, 19, 20, 22-60, 62-65, 67], 11 were machine learning algorithms $[12,18,26,28,54$, $61,67,68]$, two were based on univariate analysis [11, 21 , and one was unclear on the method of derivation [66] (Table 2). Within the regression based tools, 51 were newly derived $[13-17,19,20,22-41,44-51,53-$ $60,63-65,67]$ and seven were model updates $[10,42$, $43,46,52,62]$. Newly derived models preferentially used automated methods (forward/backward stepwise) for predictor selection during multivariate modelling (reported in 30 derivation procedures $[14-17,19,20,22$, $23,25,26,28-31,33,35-37,40,41,44,46,48,49,51$, $54,56,63-65])$, whereas the method was unclear for ten $[13,24,32,38,45,55,57,58,60]$. Model updates consisted in intercept recalibrations [42], modifications of cut-off levels for specific variables [10], and removal or introduction of new variables $[43,46,52,62]$. Of the 58 regression based tools found, more than half (32) were presented as scores $[10,11,13,14,16,19,25,34-36$, $38-40,43,45,46,48,49,51,52,56-58,60,62-64,66]$. Machine learning methods employed were artificial neural networks used in four tools $[26,28,54,68]$, decision trees used in four tools [12, 18, 67], support vector machine used in one tool [54], random forests used in one tool [54], and a hybrid approach (decision tree + support vector machine) also used in one tool [61] (Table 1). Internal validation methods were used in 19 derivations: bootstrapping was used in two [58, 59], cross-validation was used in ten $[35,38,54,63,67,68]$ and split sample was used in seven $[13,16,26,51,56$, $57,61]$.

\section{Prognostic tool performance}

C-statistics and respective 95\% confidence intervals were retrieved from 38 mortality prediction tools and 15 functional outcome prediction tools (Table 1). Forest plots are depicted in Figs. 3 and 4. The lowest reported value was 0.745 [49] and the highest reported value was 0.984 [28]. Table 3 depicts robust variance estimates of pooled c-statistics for all tools combined and subgroup analysis for mortality prediction tools, functional outcome prediction tools, logistic regression based tools, and machine learning algorithms, along with comparisons using metaregression. All subgroups showed values for pooled c statistics $>0.80$. Mortality prediction tools and machine learning algorithms showed higher pooled AUCs but the differences were not statistically significant.
Other measures of discrimination reported include accuracy, reported for 22 tools [20, 24, 26-30, 33, 37, 40-42, $54-56,63,68]$, sensitivity and/or specificity, reported for 31 tools [10, 14, 16, 22, 24, 31, 34, 38, 39, 41, 43, 44, 46, $49-51,54-56,58,62,63,68]$, and predictive values, reported for 22 tools [10, 14, 31, 38, 41, 43, 44, 46, 51, 52, 54, 56, 58, 62, 63]. Calibration assessment was reported using a calibration plot for three derivations $[42,59,64]$, the Hosmer-Lemeshow test for 14 derivations [20, 22, 24, 28, 32, 33, 39, 47, 51, 58, 60, 63] and the Le Cessie and Howelingen test reported for one derivation [64].

\section{Discussion}

Prognostic models for $\mathrm{ICH}$ patients have demonstrated good discrimination in derivation studies, regardless of the outcome in question (mortality or functional outcome). These tools have been derived in different $\mathrm{ICH}$ populations, ranging from "general" ICH (i.e. primary or spontaneous) to more specific populations (ex. arteriovenous malformation related bleeds, dialysis patients, comatose patients). Cohort studies are the predominant study design: this design is well suited for prognostic tool derivations due to an optimal measurement of predictors and outcome [69]. Other sources of data used included registries, case-control studies, randomized clinical trial data and administrative databases. Of these, the last two raise concerns about representativeness and quality of data: on one side, clinical trials usually have the highest quality of data, but restrictive inclusion and exclusion criteria might hamper generalizability [70]; on the other side, administrative databases might allow for easy access to a large quantity of patient data, but they are prone to errors in codification, data discrepancy, and missing data [71]. A considerable number of studies $(n$ =11) were multicentric, conceding a theoretical advantage in terms of generalizability. The sampling method was frequently not reported $(n=15)$ but was consecutive for most studies, again assuring the representativeness of the population and minimizing in a convenient manner the risk of bias due to selective sampling.

Most mortality prediction tools focused on death at discharge or 1 month: this timing seems appropriate, since most deaths due to $\mathrm{ICH}$ occur early in the disease [1]. However, the same cannot be said for functional outcome prediction: significant changes in functional status have been described in ICH patients up to 1 year [72], rendering outcome predictions at 1 month or discharge less useful. Noticeably, 12 derivation procedures focused on functional outcome at discharge or 1 month. A reasonable compromise would be prediction at three to 6 months, allowing enough time for patient recovery without excessive loss to follow up or occurrence of competing events. Another important issue is that studies 


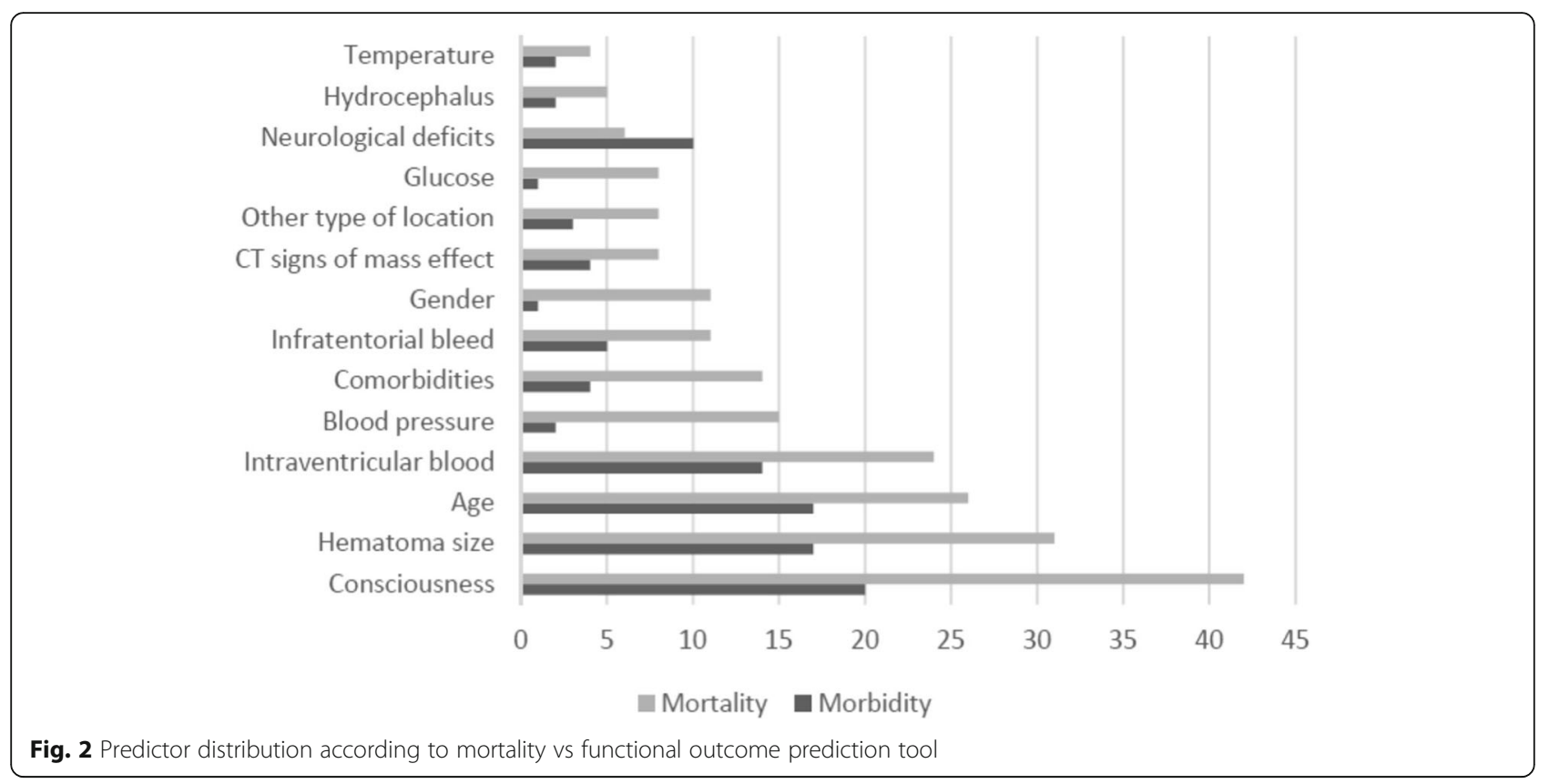

with longer follow-ups did not report on outpatient care interventions (ex. rehabilitation), making generalizability of their results less straightforward. Functional outcome prediction was mostly binary and used different scales and cut off values: whereas the optimal method of functional outcome measurement in $\mathrm{ICH}$ patients is debatable [73], the usage of different scales and cut offs between tool derivation studies makes comparisons between these instruments more difficult. Only five studies reported blinded outcome assessment: whereas mortality is a rather "hard outcome", functional outcome

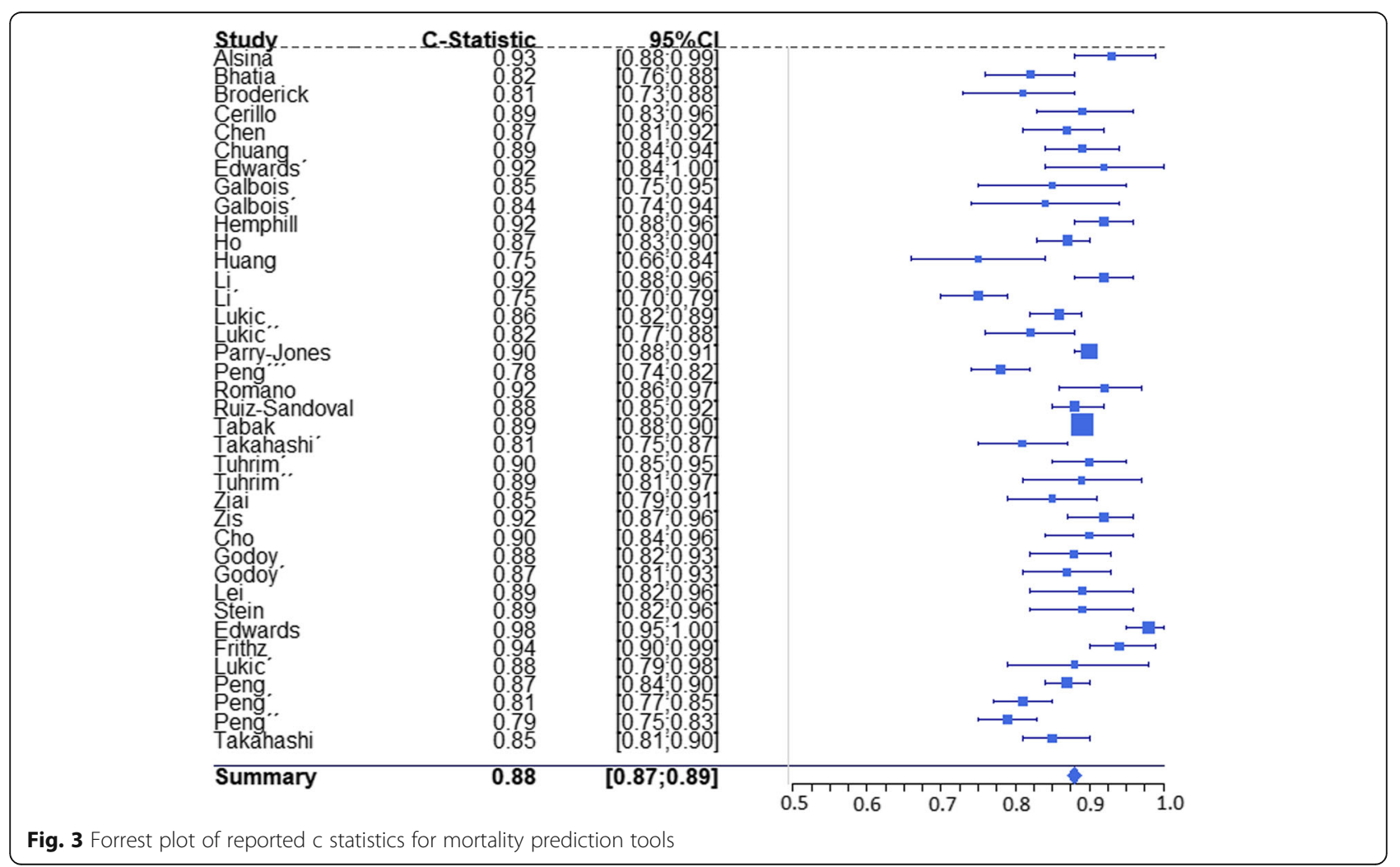




\begin{tabular}{|c|c|c|c|c|}
\hline & Studdy & C-Statistic & $95 \% \mathrm{Cl}$ & \\
\hline & Appelboom & 0.91 & {$[0.84 ; 0.99]$} & $\longmapsto$ \\
\hline & Creutzfeld & 0.93 & {$[0.90 ; 0.96]$} & $\mathrm{H}-\mathrm{H}$ \\
\hline & Hallevy & 0.90 & {$[0.85 ; 0.94]$} & $\mapsto$ \\
\hline & $\mathrm{Ji}$ & 0.84 & {$[0.82 ; 0.85]$} & $\square$ \\
\hline & Neidert & 0.84 & {$[0.75 ; 0.93]$} & $\longmapsto$ \\
\hline & Rost & 0.88 & {$[0.85 ; 0.91]$} & 늘- \\
\hline & Weimar & 0.81 & {$[0.77 ; 0.85]$} & 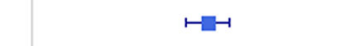 \\
\hline & Weimar & 0.86 & {$[0.80 ; 0.92]$} & $\longmapsto$ \\
\hline & Weimar" & 0.91 & {$[0.87 ; 0.94]$} & $\mapsto$ \\
\hline & Godoy & 0.89 & {$[0.84 ; 0.94]$} & $\mapsto$ \\
\hline & Godoy & 0.90 & {$[0.85 ; 0.94]$} & •ロ- \\
\hline & Lei & 0.81 & {$[0.75 ; 0.87]$} & $\longmapsto$ \\
\hline & Stein & 0.85 & {$[0.74 ; 0.96]$} & $\longmapsto$ \\
\hline & Flemming & 0.89 & {$[0.80 ; 0.98]$} & $\longmapsto$ \\
\hline & Flemming & 0.92 & {$[0.86 ; 0.98]$} & $\longmapsto$ \\
\hline & Summary & 0.87 & {$[0.84 ; 0.90]$} & " \\
\hline & & & & $\begin{array}{llllll}0.5 & 0.6 & 0.7 & 0.8 & 0.9 & 1.0\end{array}$ \\
\hline
\end{tabular}

evaluation is inherently more subjective and thus more prone to evaluation bias.

Derivation studies were rather heterogeneous in the number of patients and events analyzed. Interestingly, the four most frequently included variables for mortality prediction were also the four most frequently included variables for functional outcome prediction (Fig. 2). This overlap suggests that mortality prediction tools should, at least to some extent, predict functional outcome and vice-versa. The number of events per variable is a simple rule of thumb to assess the adequacy of sample size: it is suggested that a minimum of ten events per variable are required to prevent overfitting during statistical modelling [73], but a lower rate was found for 21 tools, although admittedly not all of them were regression based.

Missing data, whether pertaining to missing predictors or loss to follow-up, is also a potential source of bias for derivation studies, with the risk of bias relating to the amount of missing data and the extent to which it is missing at random. Handling of missing predictors was frequently not reported (22 studies). Where it was reported, complete case-analysis was the method most frequently used, which potentially creates non-random, non-representative samples of the source population. For this purpose, guidelines for prediction modelling studies have suggested preferential use of other methods such as multiple imputation, noticing however that if the number of missing predictors is extensive this technique will not be sufficient to handle this problem [69]. The same argument regarding risk of bias may be made for loss to follow-up: 4 studies reported a loss to follow-up > 10\%.

Discrimination and calibration are important properties for predictive models that should be reported. Discrimination relates to the extent to which a model distinguishes those who will suffer the outcome of interest from those who will not, whereas calibration refers to the agreement between observed and predicted outcome rates [74]. C statistic is the most commonly used performance measure for discrimination [75] but it was retrieved for only 38 derivations focusing on mortality and 15 derivations focusing on functional outcome. Taken together, these studies demonstrated good discriminatory ability for both predictions. The pooled C statistic for mortality prediction was 0.880 and the pooled $\mathrm{C}$ statistic for functional outcome prediction was 0.872 but these results must be interpreted with caution, due to the heterogeneity in the included studies in terms of population studied, selected predictors, method of model development and choice of outcome. Other forms of discriminatory ability reported include accuracy, sensitivity/ specificity, and positive/negative predictive values, but the interpretation of these measurements is less straightforward: the first two require the use of cut-off points for predicted probabilities, therefore not allowing the full use of model information, whereas the last depend on the overall probability of the event in the studied sample, hampering extrapolations for other populations with different event rates. Calibration was only reported for 14 tools, either using the Hosmer-Lemeshow test, the Le Cessie and Howelingen test, or a calibration plot.

The most frequently used method for model derivation was logistic regression. There seems to be no consensus about the best method for variable selection during multivariate logistic regression modelling, but most studies used automatic methods. These methods allow for a more efficient use of data but come with an added risk of model overfit and possible exclusion of important predictor variables due to chance, especially 
Table 3 RVE pooled c statistics and subgroup comparisons using metaregression

\begin{tabular}{|c|c|c|c|c|c|c|c|c|c|c|}
\hline \multirow[t]{2}{*}{ Prognostic tools } & \multirow{2}{*}{$\begin{array}{l}\mathrm{Nr} \\
\text { studies }\end{array}$} & \multirow{2}{*}{$\begin{array}{l}\mathrm{Nr} \\
\text { tools }\end{array}$} & \multirow{2}{*}{$\begin{array}{l}\text { Pooled } \\
\text { c-stat }\end{array}$} & \multicolumn{2}{|l|}{$95 \% \mathrm{Cl}$} & \multirow[t]{2}{*}{$1^{2}$} & \multirow[t]{2}{*}{ B } & \multicolumn{2}{|l|}{$95 \% \mathrm{Cl}$} & \multirow[t]{2}{*}{$\mathrm{p}$} \\
\hline & & & & Lower & Upper & & & Lower & Upper & \\
\hline Overall & 40 & 53 & 0.878 & 0.864 & 0.891 & $79 \%$ & - & - & - & - \\
\hline Mortality prediction tools & 30 & 38 & 0.880 & 0.865 & 0.894 & $80 \%$ & $-0.007^{\mathrm{a}}$ & $-0.039^{a}$ & $0.026^{a}$ & 0.679 \\
\hline Functional outcome prediction tools & 13 & 15 & 0.872 & 0.842 & 0.901 & $77 \%$ & & & & \\
\hline Logistic regression based tools & 37 & 43 & 0.874 & 0.858 & 0.889 & $76 \%$ & $0.018^{b}$ & $-0.034^{b}$ & $0.070^{\mathrm{b}}$ & 0.490 \\
\hline Machine learning algorithms & 6 & 9 & 0.898 & 0.821 & 0.976 & $88 \%$ & & & & \\
\hline
\end{tabular}

${ }^{a}$ mortality prediction tools as reference group

${ }^{b}$ logistic regression based tools as reference group

when sample sizes are small [76]. Nearly half of the regression based tools were simplified in the form of risk scores, allowing for an easier application. Machine learning algorithms found in our systematic review included decision trees (four), artificial neural networks (four), support vector machines (one), random forests (one) and a hybrid approach (one). These methods are an alternative to logistic regression that requires less formal statistical training and offer more efficient use of data and a higher ability to detect non-linear relations. However, they are prone to overfitting, extremely sensible to small perturbations in data and empirical in the nature of model development [77, 78]. Despite being pointed as more statistically efficient, these methods were not superior to logistic regression for discrimination in our review.

When models are tested in the same sample on which they were derived, their results tend to be biased due to overfitting: to minimize this problem, internal validation (resampling) techniques can be used. Only 19 derivations used resampling techniques for overfit adjustment. Bootstrapping is recommended as the preferred method of internal validation [74], but was performed for only two. Other methods encountered included cross-validation and split sample. The later, used in three tools, is regarded as the least effective method since it reduces statistical power for the derivation procedure and does not validate the results in a new population.

In summary, the results from our review suggest that the most promising prognostic tools are i) logistic regression based risk scores, which combine the high discrimination showed by logistic regression with the ease of application typical of prognostic scores; ii) derived from general cohorts (i.e, spontaneous or primary $\mathrm{ICH}$ ) to maximize generalizability; iii) without significant loss to follow up, to minimize risk of bias; iv) with early outcome measurement for mortality (i.e, discharge or 1 month) and later outcome measurement for functional outcome (i.e, 3 months or more) and v) showing high discrimination with an appropriate EPV rate. Examples of such scores include the scores by Chen [45], Hemphill [48], Ho [64], Romano [56] and Ruiz-Sandoval [58] for mortality, Ji [51] and Rost [57] for functional outcome prediction and Godoy [62] for a combined outcome. Not surprisingly, several validation studies have been published for these tools. Other factors to take into account are internal validation and blinded outcome assessment, the latter being particularly important for functional status.

Our review has limitations. Firstly, there were no clear guidelines on conducting and reporting studies for prognostic tool derivation at the time most of these studies were performed. This lead to frequent underreporting and higher difficulty in retrieving information about important methodological aspects and performance measures, which reflected on the results of our review. As an example, we were only able to retrieve c-statistics for 53 derivations, which means that several tools could not be evaluated for this important discrimination measure. Guidelines have recently been published to give guidance on this issue [69]. Second, studies have demonstrated that healthcare professionals are frequently pessimistic in the face of neurological emergencies [79]. This negative perception can result in a "self-fulfilling prophecy", whereby the physician's perception will lead to early withdrawal of care which, by itself, will facilitate a negative outcome [79]. Most studies assessing the effect of early care limitation in the performance of prognostic models have focused on validation studies [47, 80, 81]. According to these studies, models underestimate adverse outcomes in patients with early care limitation and overestimate in patients without. However, care limitation has also been demonstrated to be an independent predictor of poor outcome [34, 82]. Hence, one should expect that withdrawal of care would affect model performance also in derivation studies, but this factor was not taken in to account in the majority of studies included in this review. A possible solution for this problem is to derive prognostic models from patient populations with maximum level of care. Such approach was more recently used by Sembill and collaborators to derive the max-ICH score [83]. Third, the previously discussed aspects of prognostic tool derivation are useful to assess the risk of bias and external validity of these 
instruments, but they do not necessarily determine the way these tools will behave in clinical practice. Risk of bias does not necessarily imply existing bias, and the ultimate issue is how they behave in an independent external dataset [84]. At the time of our search we identified external validation studies for only 27 prognostic tools $[14,16,20,22,26,27,29-31,37,40,41,43-46,48,54$, 56-59, 62, 63]. Nevertheless, derivation studies less prone to bias are more likely to perform well in validation studies. The issues discussed in this systematic review should then be taken as a guidance for future studies seeking to validate existing prognostic tools or to derive new ones in $\mathrm{ICH}$ patients as well as in other populations.

\section{Conclusions}

Prognostic models showed high discrimination in derivation studies for mortality and functional outcome prediction in $\mathrm{ICH}$ patients but numerous methodological and reporting deficiencies were present, namely insufficient length of follow-up for functional outcome, absence of blinding, reporting and handling of missing data, low EPV rate, infrequent use of appropriate internal validation procedures and underreporting of important model performance measures. Machine learning methods have not proven to be superior to regression based models and a significant number of these tools weren't submitted to external validation. Guidelines have been reported to support authors in developing and reporting studies both for prognostic model derivation and validation [69].

\section{Additional file}

Additional file 1: Search syntax used for identification of candidate studies. (DOCX $1028 \mathrm{~kb}$ )

\section{Abbreviations \\ AUC: Area under the ROC curve; Cl: Confidence interval; EPV: Event per variable; ICH: Intracerebral hemorrhage; ROC: Receiver operating characteristic; RVE: Robust variance estimation}

\section{Acknowledgements}

Not applicable.

\section{Funding}

This work was partially supported by the project "NanoSTIMA" (NORTE-010145-FEDER-000016), which is financed by the North Portugal Regional Operational Program (NORTE 2020), under the PORTUGAL 2020 Partnership Agreement, and through the European Regional Development Fund (ERDF) The funding source had no role in the design of the study, data collection, analysis, interpretation or writing of the manuscript.

\section{Availability of data and materials}

The datasets used and/or analyzed during the current study are available from the corresponding author on reasonable request.

\section{Authors' contributions}

TG and LA designed the study; TG, SP, GA, PC and IA reviewed all references (scan read) and studies (full-text read) and gathered information and data; TG performed statistical analysis and all authors participated in the interpretation; LA and PCC oversaw the execution of the study; all authors reviewed and approved the final version of the manuscript.

Ethics approval and consent to participate

Not applicable.

\section{Consent for publication}

Not applicable.

\section{Competing interests}

The authors declare that they have no competing interests.

\section{Publisher's Note}

Springer Nature remains neutral with regard to jurisdictional claims in published maps and institutional affiliations.

\section{Author details}

'Department of Internal Medicine, Vila Nova de Gaia Hospital Cente, Rua Conceição Fernandes, 4434-502 Vila Nova de Gaia, Portugal. ${ }^{2}$ Stroke Unit, Vila Nova de Gaia Hospital Center, Rua Conceição Fernandes, 4434-502 Vila Nova de Gaia, Portugal. ${ }^{3}$ Intensive Care Department, Algarve University Hospital Center, Rua Leão Penedo, 8000-386 Faro, Portugal. ${ }^{4}$ Department of Internal Medicine, São João Hospital Center, Alameda Prof. Hernani Monteiro, 4200-319 Porto, Portugal. ${ }^{5}$ Stroke Unit, São João Hospital Center, Alameda Prof. Hernani Monteiro, 4200-319 Porto, Portugal. ${ }^{6}$ Department of Surgery and Physiology, Faculty of Medicine, University of Porto, Alameda Prof. Hernani Monteiro, 4200-319 Porto, Portugal. ${ }^{7}$ Center for Health Technology and Services Research \& Department of Community Medicine, Information and Health Decision Sciences, Faculty of Medicine, University of Porto, Alameda Prof. Hernani Monteiro, 4200-319 Porto, Portugal.

Received: 28 June 2018 Accepted: 7 November 2018

Published online: 20 November 2018

\section{References}

1. Van Asch CJJ, Luitse MJA, Rinkel GJE, Van Der Tweel I, Algra A, Klijn CJM. Incidence, case fatality, and functional outcome of intracerebral haemorrhage over time, according to age, sex , and ethnic origin : a systematic review and meta-analysis. Lancet Neurol. 2017;9(2):167-76.

2. Hemphill JC, Greenberg SM, Anderson CS, Becker K, Bendok BR, Cushman $\mathrm{M}$, et al. Guidelines for the Management of Spontaneous Intracerebral Hemorrhage: a guideline for healthcare professionals from the American Heart Association/American Stroke Association. Stroke. 2015;46:2032-60.

3. Steiner T, Salman RA, Beer R, Christensen H, Cordonnier C, Csiba L, et al. Guidelines European stroke organisation ( ESO ) guidelines for the management of spontaneous intracerebral hemorrhage. Int J Stroke. 2014: 9(7):840-55

4. Hwang BY, Appelboom G, Kellner CP, Carpenter AM, Kellner MA, Gigante PR, et al. Clinical grading scales in intracerebral hemorrhage. Neurocrit Care. 2010;13(1):141-51.

5. Moons KGM, de Groot JAH, Bouwmeester W, Vergouwe Y, Mallett S, Altman DG, et al. Critical appraisal and data extraction for systematic reviews of prediction modelling studies: the CHARMS checklist. PLoS Med. 2014;11(10): e1001744

6. Moher D, Liberati A, Tetzlaff J, Altman DG, Altman D. Preferred reporting items for systematic reviews and meta-analyses: the PRISMA statement. PLoS Med. 2009;6(7):e1000097.

7. Stroup DF, Berlin JA, Morton SC, Olkin I, Williamson GD, Rennie D, et al. Meta-analysis of observational studies in epidemiology a proposal for reporting. JAMA. 2000;283(15):2008-12.

8. Hanley AJ, McNeil JB. The meaning and use of the area under a receiver operating characteristic (ROC) curve. Radiology. 1982;143:29-36.

9. Tanner-Smith EE, Tipton E. Robust variance estimation with dependent effect sizes: practical considerations including a software tutorial in Stata and spss. Res Synth Methods. 2014;5(1):13-30.

10. Appelboom G, Hwang BY, Bruce SS, Piazza MA, Kellner CP, Meyers PM, et al. Predicting outcome after arteriovenous malformation-associated 
intracerebral hemorrhage with the original ICH score. World Neurosurg. 2012;78(6):646-50.

11. Neidert MC, Lawton MT, Mader M, Seifert B, Valavanis A, Regli L, et al. The AVICH score: a novel grading system to predict clinical outcome in arteriovenous malformation-related intracerebral hemorrhage. World Neurosurg. 2016;92:292-7.

12. Fritz G, Werner I. Studies on cerebrovascular strokes. II. Clinical findings and short-term prognosis in a stroke mater. Acta Med Scand. 1976;199:133-40.

13. Lei C, Wu B, Liu M, Zhang S, Yuan R. Cerebral amyloid Angiopathy-related intracerebral hemorrhage score for predicting outcome. Curr Neurovasc Res. 2016;13(2):156-62.

14. Cho D-Y, Chen C-C, Lee W-Y, Lee H-C, Ho L-H. A new modified intracerebral hemorrhage score for treatment decisions in basal ganglia hemorrhage--a randomized trial. Crit Care Med. 2008;36(7):2151-6.

15. Misra UK. A multivariate analysis of prognostic predictors of putaminal haemorrhage. Indian J Med Res. 1999;109:67-70.

16. Stein M, Luecke M, Preuss M, Boeker DK, Joedicke A, Oertel MF. Spontaneous intracerebral hemorrhage with ventricular extension and the grading of obstructive hydrocephalus: the prediction of outcome of a special life-threatening entity. Neurosurgery. 2010;67(5):1243-51.

17. Shah SD, Kalita J, Misra UK, Mandal SK, Srivastava M. Prognostic predictors of thalamic hemorrhage. J Clin Neurosci. 2005;12(5):559-61.

18. Flemming KD, Wijdicks EFM, Li H. Can we predict poor outcome at presentation in patients with lobar hemorrhage? Cerebrovasc Dis. 2001;11(3):183-9.

19. Shaya M, Dubey A, Berk C, Gonzalez-Toledo E, Zhang J, Caldito G, et al. Factors influencing outcome in intracerebral hematoma: a simple, reliable, and accurate method to grade intracerebral hemorrhage. Surg Neurol. 2005; 63(4):343-8.

20. Portenoy RK, Lipton RB, Berger AR, Lesser ML, Lantos G. Intracerebral haemorrhage: a model for the prediction of outcome. J Neurol Neurosurg Psychiatry. 1987;50(8):976-9.

21. Cerillo A, Vizioli L, Falivene R, Mottolese C, Bernini FP, Tedeschi G. Intracerebral hemorrhage. An attempt of statistical assessment for operability. Acta Neurol (Napoli). 1981;3(4):572-86.

22. Lisk DR, Pasteur W, Rhoades H, Putnam RD, Grotta J. Early presentation of hemispheric intracerebral hemorrhage: predictors of outcome and guidelines for treatment allocation. Neurology. 1994;44:133-9.

23. Tuhrim S, Horowitz D, Sacher M, Godbold J. Volume of ventricular blood is an important determinant of outcome in supratentorial intracerebral hemorrhage. Crit Care Med. 1999;27(3):617-21.

24. Alsina AE. Hemorragia supratentorial espontánea : un modelo matemático predictivo de mortalidad. Salud(i)Ciencia. 2014;20(i):368-72.

25. Hallevy C, Ifergane G, Kordysh E, Herishanu Y. Spontaneous supratentorial intracerebral hemorrhage: criteria for short-term functional outcome prediction. J Neurol. 2002;249(12):1704-9.

26. Lukic S, Cojbasic Z, Peric Z, Spasic M. Comparison of artificial neural network and regression analysis for prediction of mortality in spontaneous intracerebral hemorrhage based on initial clinical parameters. HealthMED. 2012;6(2):375-81.

27. Masè G, Zorzon M, Biasutti E, Tasca G, Vitrani B, Cazzato G. Immediate prognosis of primary intracerebral hemorrhage using an easy model for the prediction of survival. Acta Neurol Scand. 1995;91:306-9.

28. Edwards DF, Hollingsworth H, Zazulia A, Diringer M. Artificial neural networks improve the prediction of mortality in intracerebral hemorrhage. Neurology. 1999;53(2):351-7.

29. Tuhrim S, Dambrosia JM, Price TR, Mohr JP, Wolf PA, Heyman A, et al. Prediction of intracerebral hemorrhage survival. Ann Neurol. 1988;24(2):258-63.

30. Tuhrim S, Dambrosia J, Price T, Mohr J, Wolf P, Hier D, et al. Intracerebral hemorrhage: external validation and extension of a model for prediction of 30-day survival. Ann Neurol. 1991;29(6):658-63.

31. Fogelholm R, Avikainen S, Murros K. Prognostic value and determinants of first-day mean arterial pressure in spontaneous Supratentorial intracerebra hemorrhage. Stroke. 1997;28(7):1396-400.

32. Szepesi R, Széll IK, Hortobágyi T, Kardos L, Nagy K, Lánczi LI, et al. New Prognostic Score for the Prediction of 30-Day Outcome in Spontaneous Supratentorial Cerebral Haemorrhage. 2015;2015:961085.

33. Lukić S, Ćojbasić Ž, Perić Z, Milošević Z, Spasić M, Pavlović V, et al. Artificial neural networks based early clinical prediction of mortality after spontaneous intracerebral hemorrhage. Acta Neurol Belg. 2012;112(4):375-82.

34. Hallevi H, Dar NS, Barreto AD, Morales MM, Abraham AT, Walker KC, et al. The IVH Score: A novel tool for estimating intraventricular hemorrhage volume: Clinical and research implications. Crit Care Med. 2009;(3):1-15.
35. Ziai WC, Siddiqui AA, Ullman N, Herrick DB, Yenokyan G, Mcbee N, et al. Early therapy intensity level ( TIL ) predicts mortality in spontaneous intracerebral hemorrhage. Neurocrit Care. 2015;23(2):188-97.

36. Tshikwela ML, Longo-Mbenza B. Spontaneous intracerebral hemorrhage: clinical and computed tomography findings in predicting in-hospital mortality in Central Africans. J Neurosci Rural Pract. 2012;3(2):115-20.

37. Weimar C, Roth M, Willig V, Kostopoulos P, Benemann J, Diener HC. Development and validation of a prognostic model to predict recovery following intracerebral hemorrhage. J Neurol. 2006;253(6): 788-93.

38. Galbois A, Boëlle PY, Hainque E, Raynal M, Cazejust J, Baudel JL, et al. Prediction of evolution toward brain death upon admission to ICU in comatose patients with spontaneous intracerebral hemorrhage using simple signs. Transpl Int. 2013;26(5):517-26.

39. Zis $P$, Leivadeas $P$, Michas $D$, Kravaritis $D$, Angelidakis $P$, Tavernarakis $A$. Predicting 30-day case fatality of primary inoperable intracerebral hemorrhage based on findings at the emergency department. I Stroke Cerebrovasc Dis. 2014;23(7):1928-33.

40. Huang BR, Liao CC, Huang WH, Hsu YH, Hsu JC, Yen HC, et al. Prognostic factors of spontaneous intracerebral haemorrhage in haemodialysis patients and predictors of 30-day mortality. Intern Med J. 2008;38(7):568-74.

41. Berwaerts J, Dijkhuizen RS, Robb OJ, Webster J. Prediction of functional outcome and in-hospital mortality after admission with oral anticoagulantrelated intracerebral hemorrhage. Stroke. 2000;31(11):2558-62.

42. Weimar C, Ziegler A, Sacco RL, Diener HC, König IR. Predicting recovery after intracerebral hemorrhage--an external validation in patients from controlled clinical trials. J Neurol. 2009;256(3):464-9.

43. Weimar C, Benemann J, Diener H-C, German stroke study collaboration. Development and validation of the Essen intracerebral Haemorrhage score. J Neurol Neurosurg Psychiatry. 2006;77(5):601-5.

44. Broderick J, Brott T, Duldner J, Tomsick T, Huster G. Volume of intracerebral hemorrhage a powerful and easy-to-use predictor of 30-day mortality. Stroke. 1993;24(7):987-93.

45. Chen HS, Hsieh CF, Chau TT, Yang CD, Chen YW. Risk factors of in-hospital mortality of intracerebral hemorrhage and comparison of $\mathrm{ICH}$ scores in a Taiwanese population. Eur Neurol. 2011;66(1):59-63.

46. Cheung RTF, Zou LY. Use of the original, modified, or new intracerebral hemorrhage score to predict mortality and morbidity after intracerebral hemorrhage. Stroke. 2003;34(7):1717-22.

47. Creutzfeld CJ, Becker KJ, Weinstein JR, Khot SP, McPharlin TO, Ton TG, et al. Do-not-attempt-resuscitation orders and prognostic models for Intraparenchymal hemorrhage. Crit Care Med. 2011;39(1):158-62.

48. Hemphill JC, Bonovich DC, Besmertis L, Manley GT, Johnston SC. The ICH score. Stroke. 2001;32:891-7.

49. Li F, Chen H, Wang Y, Yang Y, Li C, Dong Z. A new prognostic model and score to predict short term outcome after intracerebral hemorrhage. Sci Res Essays. 2011;6(10):2063-8.

50. Li Y-F, Luo J, Li Q, Jing Y-J, Wang R-Y, Li R-S. A new simple model for prediction of hospital mortality in patients with intracerebral hemorrhage. CNS Neurosci Ther. 2012;18(6):482-6.

51. Ji R, Shen H, Pan Y, Wang P, Liu G, Wang Y, et al. A novel risk score to predict 1-year functional outcome after intracerebral hemorrhage and comparison with existing scores. Crit Care. 2013;17(6):R275.

52. Mittal MK, Lele A. Predictors of poor outcome at hospital discharge following a spontaneous intracerebral hemorrhage. Int J Neurosci. 2011;121(5):267-70.

53. Parry-Jones AR, Abid KA, Di Napoli M, Smith CJ, Vail A, Patel HC, et al. Accuracy and clinical usefulness of intracerebral hemorrhage grading scores: a direct comparison in a UK population. Stroke. 2013;44(7):1840-5.

54. Peng S, Chuang Y, Kang T, Tseng K. Random forest can predict 30-day mortality of spontaneous intracerebral hemorrhage with remarkable discrimination. Eur J Neurol. 2010;17:945-50.

55. Poungvarin N, Suwanwela NC, Venketasubramanian N, Wong LKS, Navarro JC, Bitanga E, et al. Grave prognosis on spontaneous intracerebral haemorrhage: GP on stage score. J Med Assoc Thail. 2006;89(5):84-93.

56. Romano LM, loli PL, Gonorazky SE, Rauek S, Santamarina RD, Besocke AG, et al. Desarollo y validación de la escala predictiva de mortalidad (REC-HPC) en la hemorragia intracerebral primaria. Neurol Argentina. 2009;1(2):75-81.

57. Rost NS, Smith EE, Chang Y, Snider RW, Chanderraj R, Schwab K, et al. Prediction of functional outcome in patients with primary intracerebral hemorrhage: the FUNC score. Stroke. 2008;39(8):2304-9. 
58. Ruiz-Sandoval JL, Chiquete E, Romero-Vargas S, Padilla-Martínez JJ, González-Cornejo S. Grading scale for prediction of outcome in primary intracerebral hemorrhages. Stroke. 2007;38(5):1641-4.

59. Tabak YP, Johannes RS, Silber JH. Using automated clinical data for risk adjustment. Med Care. 2007;45(8):789-805.

60. Safatli DA, Gunther A, Schlattmann P, Schwarz F, Kalff R, Ewald C. Predictors of 30-day mortality in patients with spontaneous primary intracerebral hemorrhage. Surg Neurol Int. 2016;7(8):S510-7.

61. Chiu H, Chiu D, Lee Y, Wang C, Wang C. To explore intracerebral hematoma with a hybrid approach and combination of discriminative factors. Methods Inf Med. 2016:55(5):450-4.

62. Godoy DA, Piñero G, Di Napoli M. Predicting mortality in spontaneous intracerebral hemorrhage: can modification to original score improve the prediction? Stroke. 2006;37(4):1038-44.

63. Chuang YC, Chen YM, Peng SK, Peng SY. Risk stratification for predicting 30-day mortality of intracerebral hemorrhage. Int J Qual Heal Care. 2009;21(6):441-7.

64. Ho WM, Lin JR, Wang HH, Liou CW, Chang KC, Der Lee J, et al. Prediction of in - hospital stroke mortality in critical care unit. Springerplus. 2016;5(1051):1-9.

65. Bhatia R, Singh H, Singh S, Padma MV, Prasad K, Tripathi M, et al. A prospective study of in-hospital mortality and discharge outcome in spontaneous intracerebral hemorrhage. Neurol India. 2013;61(3):244-8.

66. Grellier P, Duplay J, Roche J, Legout A, Miramond A. Proposition pour une évaluation pronostique chiffrée dans l'hématome intra-cérébral. Neurochirurgie. 1983;29(4):261-2.

67. Takahashi O, Cook EF, Nakamura T, Saito J, Ikawa F, Fukui T. Risk stratification for in-hospital mortality in spontaneous intracerebral haemorrhage: a classification and regression tree analysis. QJM. 2006;99(11):743-50.

68. Çelik G, Baykan O, Kara Y, Tireli H. Predicting 10-day mortality in patients with strokes using neural networks and multivariate statistical methods. J Stroke Cerebrovasc Dis. 2014;23(6):1506-12.

69. Moons KGM, Altman DG, Reitsma JB, loannidis JPA, Macaskill P, Steyerberg EW, et al. Transparent Reporting of a multivariable prediction model for Individual Prognosis Or Diagnosis (TRIPOD): Explanation and Elaboration. Ann Intern Med. 2015;162:W1-73.

70. Booth CM, Tannock IF. Randomised controlled trials and population-based observational research: partners in the evolution of medical evidence. $\mathrm{Br} J$ Cancer. 2014;110(3):551-5.

71. Schlussel AT, Delaney CP, Maykel JA, Lustik MB, Nishtala M, Steele SR. A National Database Analysis Comparing the Nationwide inpatient sample and American College of Surgeons National Surgical Quality Improvement Program in laparoscopic vs open colectomies: inherent variance may impact outcomes. Dis Colon Rectum. 2016;59(9):843-54.

72. Hemphill JC, Farrant M, Neill TA. Prospective validation of the ICH score for 12-month functional outcome. Neurology. 2009;73(14):1088-94.

73. Quinn T, Harrison KS, McArthur TJ. Assessment scales in stroke: clinimetric and clinical considerations. Clin Interv Aging. 2013;8:201-11.

74. Steyerberg EW, Harrell FE, Borsboom GJJ, Eijkemans MJ, Vergouwe Y, Habbema JDF. Internal validation of predictive models. J Clin Epidemiol. 2001;54(8):774-81.

75. Steyerberg EW, Vickers AJ, Cook NR, Gerds T, Obuchowski N, Pencina MJ, et al. Assessing the performance of prediction models : a framework for some traditional and novel measures. Epidemiology. 2010;21(1):128-38.

76. Austin PC, Tu JV. Automated variable selection methods for logistic regression produced unstable models for predicting acute myocardial infarction mortality. J Clin Epidemiol. 2004;57(11):1138-46.

77. Kokol P, Zorman M, Stiglic MM, Malèiae I. The limitations of decision trees and automatic learning in real world medical decision making. Stud Health Technol Inform. 1998;52(1):529-33.

78. Tu JV. Advantages and disadvantages of using artificial neural networks versus logistic regression for predicting medical outcomes. J Clin Epidemiol. 1996:49(11):1225-31

79. Becker KJ, Baxter AB, Cohen WA, Bybee HM, Tirschwell DL, Newell DW, et al. Withdrawal of support in intracerebral hemorrhage may lead to self-fulfilling prophecies. Neurology. 2001;56(6):766-72.

80. Zahuranec DB, Morgenstern LB, Sánchez BN, Resnicow K, White DB, Hemphill JC. Do-not-resuscitate orders and predictive models after intracerebral hemorrhage. Neurology. 2010;75:626-33.

81. Garrett JS, Zarghouni M, Layton KF, Graybeal D, Daoud YA. Validation of clinical prediction scores in patients with primary intracerebral hemorrhage. Neurocrit Care. 2013;19(3):329-35.
82. Ziai WC, Siddiqui SS, Ullman N, Herrick DB, Yenokyan G, Mcbee N, , et al. Early therapy intensity level ( TIL ) predicts mortality in spontaneous intracerebral hemorrhage. Neurocrit Care 2015;23(2):188-197.

83. Sembill JA, Gerner ST, Volbers B, Bobinger T, Lucking H, Kloska SP, et al. Severity assessment in maximally treated $\mathrm{ICH}$ patients: the max- $\mathrm{ICH}$ score. Neurology. 2017:89(5):423-31.

84. Altman DG, Royston P. What do we mean by validating a prognostic model? Stat Med. 2000;19(4):453-73.
Ready to submit your research? Choose BMC and benefit from:

- fast, convenient online submission

- thorough peer review by experienced researchers in your field

- rapid publication on acceptance

- support for research data, including large and complex data types

- gold Open Access which fosters wider collaboration and increased citations

- maximum visibility for your research: over $100 \mathrm{M}$ website views per year

At $\mathrm{BMC}$, research is always in progress.

Learn more biomedcentral.com/submissions 